\title{
Zap-and-Freeze Electron Microscopy Captures Synaptic Vesicle Exocytosis with Unprecedented Temporal Precision
}

\author{
Grant F. Kusick ${ }^{1,2}$, Morven Chin ${ }^{3,}$ and Shigeki Watanabe ${ }^{1,4}$ \\ 1. Department of Cell Biology, Johns Hopkins University, School of Medicine, Baltimore, MD 21205, \\ USA \\ 2. Biochemistry, Cellular and Molecular Biology Graduate Program, Johns Hopkins University, School \\ of Medicine, Baltimore, MD 21205, USA \\ 3. Department of Biology, Dartmouth College, Hanover, NH 03755, USA \\ 4. Solomon H. Snyder Department of Neuroscience, Johns Hopkins University, School of Medicine, \\ Baltimore, MD 21205, USA.
}

Neurotransmission at chemical synapses is the fundamental information processing event in the nervous system. This information transfer is discrete: action potentials trigger the release of quantal packets of neurotransmitter [1]. Heuser and Reese used quick-freezing and electron microscopy to show that each quantum is a single neurotransmitter-containing synaptic vesicle that fuses with the plasma membrane at a specialized "active zone" [2]. Intense genetic and biochemical investigation has since formed a detailed picture of the molecular basis for synaptic vesicle exocytosis [3]. However, several basic aspects of this process remain, at best, controversial and, at worst, completely unresolved.

These controversies persist mainly because the speed of synaptic vesicle exocytosis and small area in which it occurs make it difficult to directly observe under physiologically-relevant conditions, particularly at small synapses of the mammalian central nervous system. We previously developed "flash-and-freeze" to capture exocytosis and compensatory endocytosis after a single AP by electron microscopy [4]. Flash-and-freeze uses channelrhodopsin to stimulate neurons with light, followed by high-pressure freezing at the desired time point. However, the limitations of channelrhodopsin made a detailed kinetic analysis of exocytosis impossible and called our results into question.

We now present "zap-and-freeze", in which an action potential is triggered by electrical field stimulation before high-pressure freezing. We developed a field stimulation device that is charged before the sample enters the freezing chamber, then discharged by a $1 \mathrm{~ms}$ pulse of light while inside the chamber so neurons can be frozen as early as $5 \mathrm{~ms}$ after the action potential (Figure 1).

Using zap-and-freeze, we observed presumed exocytic intermediates apposed to the postsynaptic plasma membrane at various stages of collapse at synapses from cultured mouse hippocampal neurons (Figure 1A). Notably, we observed exocytic intermediates at 5, 8, and $11 \mathrm{~ms}$ after the action potential, but not at $14 \mathrm{~ms}$ (Figure 1B), placing the collapse rate of synaptic vesicles under $15 \mathrm{~ms}$, faster than previous estimates from systems with less temporal precision. Multiple vesicles fused with the plasma membrane at single synapses, a debated phenomenon that had never been directly observed. Finally, we found that vesicles fusion occurs throughout the active zone, but are strongly biased towards the periphery. These results establish fundamental parameters of synaptic vesicle exocytosis and provide a foundation for investigating the underlying molecular mechanisms.

References:

[1] JE Heuser et al., J Cell Biol. 81 (1979), p. 275-300. 
[2] J Del Castillo and B Katz, J. Physiol. 124 (1954), p. 560-573.

[3] TC Südhof, Neuron 80 (2013), p. 675-690.

[4] Watanabe et al., Nature 504 (2013), p. 242-7.

A

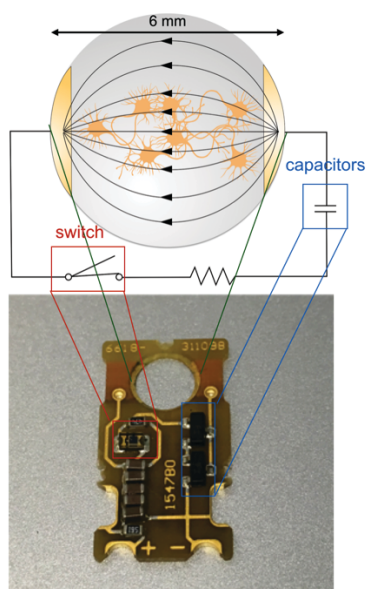

B

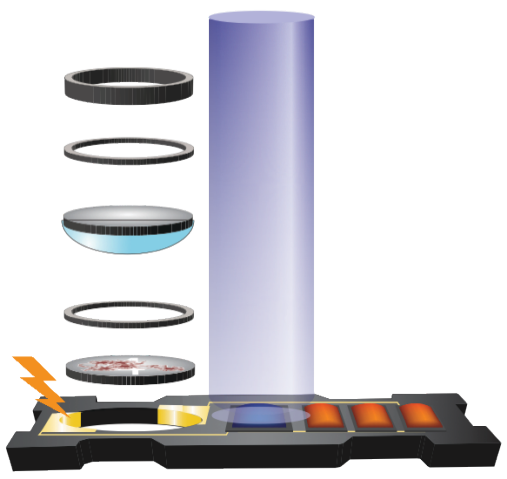

Figure 1. Zap-and-freeze device for coupling electrical field stimulation with high-pressure freezing. A) Picture of the "middle plate" device and schematic showing the principle behind stimulation. B) Illustration of the setup for zap-and-freeze.

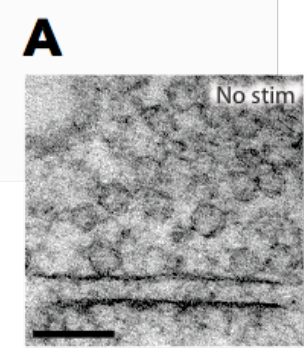

B

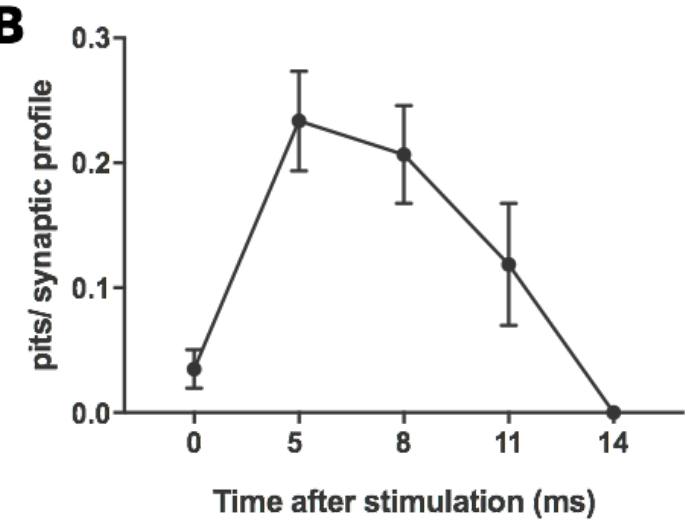

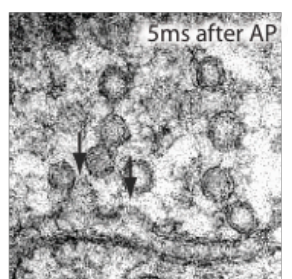

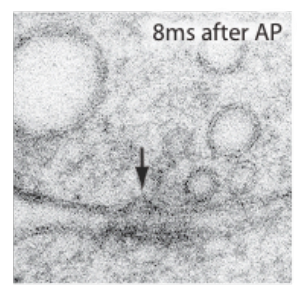

C
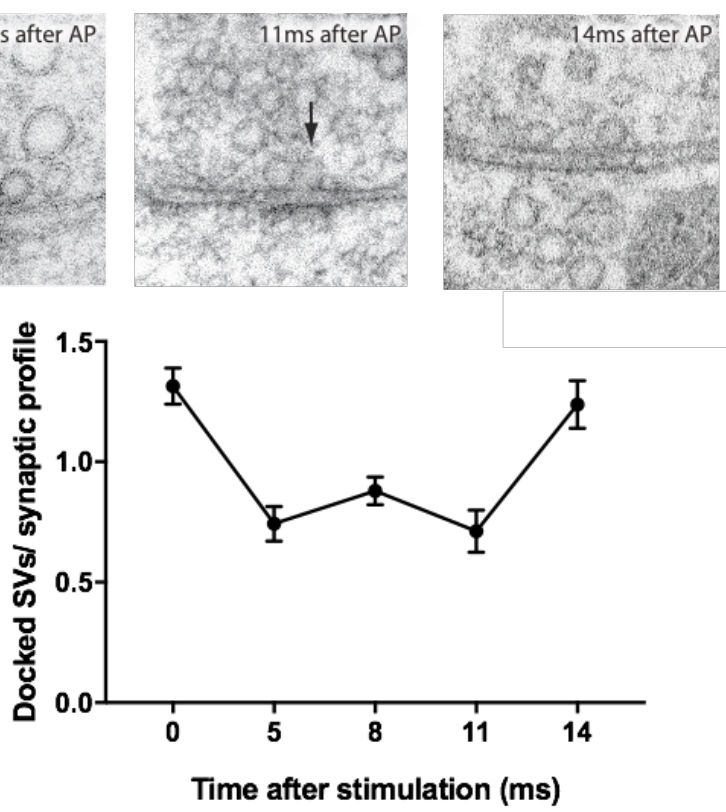

Figure 2. Time course of exocytosis at cultured mouse hippocampal synapses revealed by zap-andfreeze. A) Representative transmission electron micrographs from neurons stimulated for $1 \mathrm{~ms}$ at the indicated time points before high-pressure freezing. Arrows indicate synaptic vesicle-sized "pits" apposed to the postsynaptic membrane, which are presumed to be exocytic intermediates. B) Quantification of pits. Vesicles appear to have fully collapsed into the plasma membrane by $14 \mathrm{~ms}$. C) Quantification of docked vesicles, which are defined as appearing to be in contact with the plasma membrane. Experiments were performed at $37{ }^{\circ} \mathrm{C}$ in physiological saline containing $1.2 \mathrm{Ca}^{2+}$. Error bars represent standard error of the mean. $\mathrm{n}>66$ for each time point. Scale bar: $100 \mathrm{~nm}$. 